\title{
Numerical study of anharmonic vibrational decay in amorphous and paracrystalline silicon
}

\author{
Jaroslav Fabian \\ Institute for Theoretical Physics, Karl-Franzens University, Universitätsplatz 5, 8010 Graz, Austria \\ Joseph L. Feldman and C. Stephen Hellberg \\ Center for Computational Materials Science, Naval Research Laboratory, Washington DC 20375-5345 \\ S. M. Nakhmanson \\ Department of Physics, North Carolina State University, Raleigh, North Carolina 27695-8202 \\ (Received 14 January 2003; revised manuscript received 26 March 2003; published 10 June 2003)
}

\begin{abstract}
The anharmonic decay rates of atomic vibrations in amorphous silicon $(a-\mathrm{Si})$ and paracrystalline silicon ( $p$-Si), containing small crystalline grains embedded in a disordered matrix, are calculated using realistic structural models. The models are 1000-atom four-coordinated networks relaxed to a local minimum of the Stillinger-Weber interatomic potential. The vibrational decay rates are calculated numerically by perturbation theory, taking into account cubic anharmonicity as the perturbation. The vibrational lifetimes for $a$-Si are found to be on picosecond time scales, in agreement with the previous perturbative and classical molecular dynamics calculations on a 216-atom model. The calculated decay rates for $p$-Si are similar to those of $a$-Si. No modes in $p$-Si reside entirely on the crystalline cluster, decoupled from the amorphous matrix. The localized modes with the largest (up to 59\%) weight on the cluster decay primarily to two diffusons. The numerical results are discussed in relation to a recent suggestion by van der Voort et al. [Phys. Rev. B 62, 8072 (2000)] that long vibrational relaxation inferred experimentally may be due to possible crystalline nanostructures in some types of $a$-Si.

DOI: 10.1103/PhysRevB.67.224302

PACS number(s): $63.50 .+\mathrm{x}, 65.60 .+\mathrm{a}$
\end{abstract}

\section{INTRODUCTION}

The pioneering experiments by Dijkhuis and co-workers ${ }^{1-4}$ explored transient dynamics of excited vibrational modes in a topologically disordered materialhydrogenated amorphous silicon. In these experiments nonequilibrium vibrational states were generated during relaxation and recombination of optically excited electrons, and monitored with a probe laser (anti-Stokes Raman spectroscopy) for transient behavior. The experimental results are surprising: Scholten et al. ${ }^{1,2}$ found that at low temperatures $(2 \mathrm{~K})$ and for vibrational frequencies greater than $10 \mathrm{meV}$ (maximum frequency in $a$-Si is about $70 \mathrm{meV}$ ) vibrations decay on time scales of tens of nanoseconds. Furthermore, the higher the vibrational frequency, the slower is the decay rate. In contrast, phonons in crystalline silicon decay on time scales of tens of picoseconds ${ }^{5}$ and the decay rates increase with increasing frequency. The results of Scholten et al. were further confirmed by van der Voort et al., ${ }^{3}$ who suggested that the long lifetimes are due to the microstructure of amorphous silicon. This suggestion was tested by van der Voort et $a{ }^{4}{ }^{4}$ by measuring the vibrational decay rates of a mixed amorphous-nanocrystalline silicon, which was an amorphous hydrogenated silicon with a sizable fraction of nanocrystallites (with the diameter of $1-5 \mathrm{~nm}$ ). Even the mixed sample displayed nanosecond vibrational lifetimes, although the lifetimes appeared to decrease with increasing frequency. A hypothesis was put forward ${ }^{4}$ that the measured types of amorphous silicon contain nanoscale regions with correlated (if not ordered) atoms, which, through enhanced size quantization and localization of vibrational frequencies, inhibit anharmonic decay.
These experimental results are at odds with the known theories of anharmonic vibrational decay in disordered materials. ${ }^{6-8}$ In their so called "fracton" model, Alexander et $a l .{ }^{9}$ assumed that the majority of vibrational states in disordered systems are localized. This seemed to explain the above experimental findings since the anharmonic decay could be drastically reduced by the extremely small likelihood of the overlap between three localized modes. ${ }^{10,11}$ That the small probability of the overlap between three localized modes inhibits vibrational decay was disputed by Fabian and Allen ${ }^{6}$ who put forward a probabilistic scaling argument that the interaction between three localized modes would in fact be crucial for the anharmonic decay and cannot be neglected. Fabian later demonstrated ${ }^{12}$ the scaling argument on a onedimensional anharmonic chain with random spring constants, and similar conclusions were reached recently by Leitner in a study of heat flow in a one-dimensional glass ${ }^{13}$ and vibrational energy transfer in helices of myoglobin. ${ }^{14}$ Thus the fracton model, even if true in its premise of localization of the majority of the vibrational modes, does not explain the experiment. We note, however, that even the premise of the model is questionable, as it is in sharp contrast to what is found in finite-size realistic models of glasses, which normally exhibit localization only in a small part of the spectrum.

Numerical calculations of vibrational decay in glasses have been performed both by evaluating a perturbation formula ${ }^{6}$ and by classical molecular dynamics. ${ }^{7,8}$ Perturbation theory was applied to the problem of anharmonic decay in glasses by Fabian and Allen ${ }^{6}$ who computed the decay rates for a 216 atom model of amorphous $\mathrm{Si}$. The decay rates were found to be fractions of $\mathrm{meV}$ (that is, lifetimes are 
picoseconds), in general increasing with increasing frequency. The anharmonic lifetimes of localized modes were similar to those of the extended modes, even in the case of a model alloy $\mathrm{Si}_{x} \mathrm{Ge}_{1-x}$, where localized modes span more than a half of the spectrum and the overlap between localized states becomes important. ${ }^{6}$ Bickham and Feldman ${ }^{7}$ reported vibrational decay rates for selected modes of 216 and 4096 atom models of amorphous $\mathrm{Si}$, using classical molecular dynamics. Their results agree with the perturbative calculation, though the computed decay rates are somewhat greater due to the fact that molecular dynamics takes into account all the anharmonic interaction, while the perturbative calculation in Ref. 6 only cubic anharmonicity. In the calculation of Bickham and Feldman, a chosen vibrational mode was given a greater than average kinetic energy and was allowed to equilibrate while keeping the overall temperature constant. From the decay of the kinetic energy in time, the mode decay rate was obtained. While the advantage of molecular dynamics over perturbation theory in calculating vibrational decay rates is that the full anharmonic interaction is considered, the disadvantage is that the classical dynamics does not capture accurately the low temperature decay rates (for example, the rates computed by classical molecular dynamics vanish at zero temperature, ${ }^{7}$ while in reality they are finite due to quantum effects ${ }^{6}$ ).

The purpose of this paper is threefold: (i) To extend the previous numerical studies of perturbative anharmonic decay in homogeneous amorphous silicon $(a-\mathrm{Si})$ to a larger system, (ii) to present computational details of the numerical evaluation of anharmonic lifetimes using perturbation theory, and (iii)to calculate vibrational decay rates for a model of amorphous silicon - paracrystalline silicon $(p-\mathrm{Si})$ - that includes nanocrystallites. The larger system is a 1000-atom model of $a$-Si, prepared similarly to the previously used 216atom model. ${ }^{6}$ The calculated decay rates display smaller statistical fluctuations and agree, on average, with those of the smaller model. Studying paracrystalline silicon, a material where small crystalline grains are embedded in a disordered matrix, allows us to test the hypothesis of van der Voort ${ }^{4}$ regarding the structural origin of the anomalous long vibrational lifetimes in a mixed amorphous-nanocrystalline Si system. In our calculations we have used a 1000-atom (86 out of which belong to a single crystalline grain) model created by Nakhmanson et al. ${ }^{15}$ to simulate medium-range order in amorphous silicon. We should point out, that, although providing a more realistic subject for the verification of van der Voort's hypothesis than "regular" models for $a$-Si, this simple model is neither an exact structural match to nanocrystalline Si sample of Ref. 4 (24\% crystalline fraction and $4.5 \mathrm{~nm}$ average grain diameter, versus $\approx 10 \%$ and $1 \mathrm{~nm}$ in the model) nor can it account for various other topological defects present in real material. Still, if van der Voort's supposition were correct, we would observe inhibited decay rates of the modes which would be predominantly localized on the crystalline cluster in the model. However, we do not find any modes localized exclusively on the cluster: one of the most cluster-localized modes has only $59 \%$ weight on the cluster, and is therefore well coupled to the disordered matrix. It is not surprising that such modes have decay rates similar to other localized states.

We remark that the names amorphous and paracrystalline in reference to our models are a matter of terminological convenience rather than an attempt in classifying real materials. We refer to $a$-Si as describing a homogeneous, continuous random network of silicon atoms, while $p$-Si models are such networks filled with crystalline clusters. Real materials - which are normally termed amorphous silicon-are likely of the $p$-Si type, containing nanoscale crystallites with a distribution of sizes. ${ }^{16}$

In the following we first introduce the structural models of $a-\mathrm{Si}$ and $p-\mathrm{Si}$ and their harmonic vibrational properties, then discuss the perturbative calculation of anharmonic decay rates and present the results for the 1000-atom models of $a$-Si and $p$-Si. Finally, we discuss our results with respect to the experiment.

\section{MODELS}

Both models employed in our studies were created with similar techniques: the homogeneous model for $a$-Si was constructed using the WWW method ${ }^{17}$ and the paracrystalline model with a variation ${ }^{15}$ of the Barkema-Mousseau method. ${ }^{18}$ (For a recent review of modeling continuous random networks see Ref. 19.) The former model was studied ${ }^{20}$ for its harmonic properties within the framework of the Stillinger-Weber (SW) potential ${ }^{21}$ prior to the present work. The major difference between the methods of WWW and Barkema-Mousseau is the starting configuration used for the model construction: crystalline silicon $(c-\mathrm{Si})$ is used in the former approach and a random close packed configuration in the latter. Both models were relaxed with respect to SW prior to the decay times calculation. The densities for the two SW relaxed models are thus found to be slightly (three to four percent) less than that of the density of $c$-Si, with the density of the paracrystalline model being slightly (two percent) higher than that of the homogeneous model. Changes in the atomic positions resulting from the SW relaxation were found to be quite small. In general it is known that the SW potential produces relaxed structures that have two to three percent fivefold coordinated atoms, even if the starting structures were perfectly fourfold coordinated (which is the case for the paracrystalline model); this deviation from the perfect fourfold coordination does not noticeably alter vibrational properties of the models.

\section{HARMONIC VIBRATIONS}

In the harmonic approximation vibrational eigenfrequencies $\omega(i)$ and eigenvectors $\mathbf{e}_{a}^{i}$ are computed by diagonalizing the corresponding dynamical matrix (throughout the paper symbols $j, k$, and $l$ will represent vibrational modes, while $a, b$, and $c$ atoms). The results of numerical calculations from various groups ${ }^{22-27}$ indicate that vibrational eigenstates in glasses belong to one of four groups: ${ }^{6,28,29}$ propagons, resonant modes, diffusons, and locons. Propagons are sound waves scattered by structural disorder. Resonances are modes temporarily trapped in topological defects. Diffusons, which 


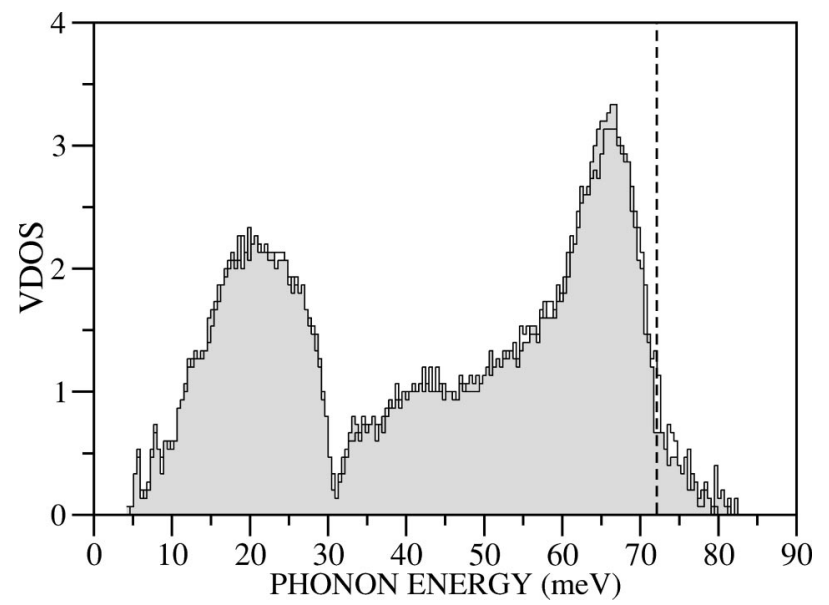

FIG. 1. Vibrational density of states of the 1000-atom models of $a$-Si (shaded area) and $p$-Si (line without shading). Modes with $\omega$ $>72 \mathrm{meV}$ (indicated by the vertical line) are locons.

make up the majority of the spectrum, and have their frequencies above the Ioffe-Regel limit ${ }^{30}$ are extended but nonpropagating modes, ${ }^{20,31}$ which cannot be labeled by momentum, only by frequency. Finally, locons are localized modes in the sense of strong (Anderson) localization. Experimentally the character of the atomic vibrations in glasses has been studied by inelastic $\mathrm{x}$-ray scattering in various glassy systems. $^{32-36}$ The recent experimental and theoretical progress is reviewed in Ref. 29.

In Fig. 1 we plot the calculated vibrational density of states (VDOS) for the models of $a$-Si and $p$-Si. Both curves look very similar, which is in agreement with the VDOS calculation of Ref. 15 made with a modified version of the SW potential. ${ }^{37}$ The calculated spectrum agrees rather well with the experimental one, ${ }^{20}$ except that the calculation overestimates the highest frequencies by about $15 \%$. This is a known artifact of the SW potential. The striking similarity

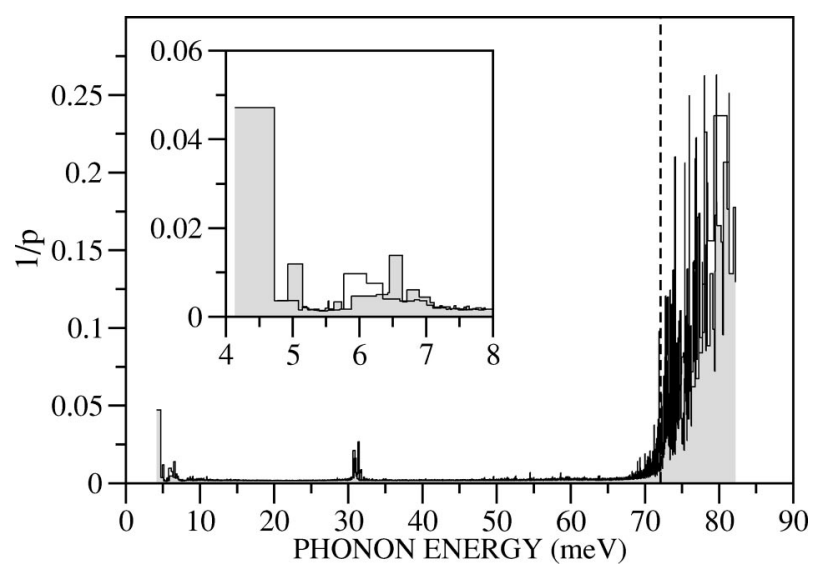

FIG. 2. Inverse participation ratio $1 / p$ of the vibrational states in the 1000-atom models of $a$-Si (shaded area) and $p$-Si (line without shading). The modes with the frequencies above $72 \mathrm{meV}$ (the vertical line) can be considered localized. Quasilocalization occurs at low frequencies (resonant modes) and around $30 \mathrm{meV}$ which corresponds to the band edges. The inset is a detailed view of the low-frequency region.

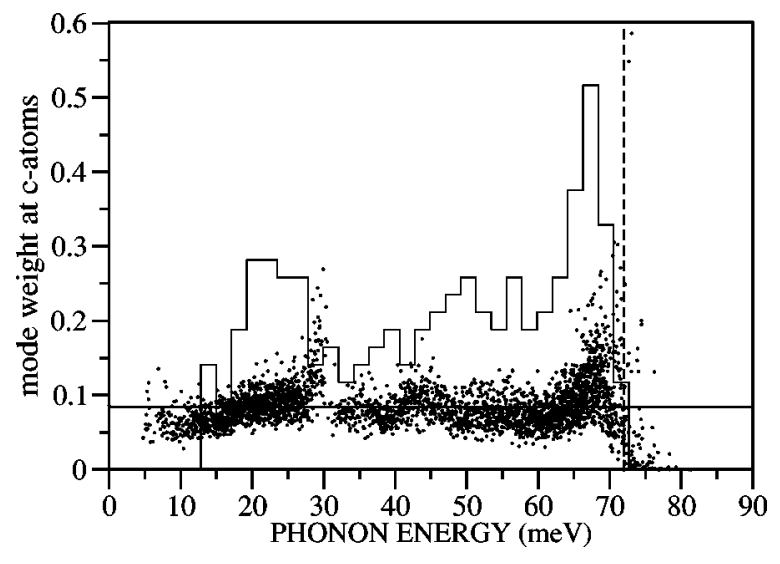

FIG. 3. Weight of the modes at the crystalline cluster as a function of mode frequency in the 1000 -atom model of $p$-Si. Plotted is the square of the atom displacement summed over the atoms forming the cluster. The horizontal line shows a weight of $0.086(8.6 \%)$ indicating an unbiased displacement pattern. The histogram is VDOS for the cluster (see text).

between VDOS of the two models suggests that the crystalline cluster does not significantly perturb the vibrational states. Experimentally, however, the presence of nanocrystals is detected as a pronounced contribution (a peak attributed to crystalline TO modes) to the Raman spectrum. ${ }^{4}$ While this may indeed suggest that the "crystalline" modes in the experimental samples are more weakly coupled to the amorphous environment, the reason for the appearance of the distinct peak in the Raman spectrum can also be the fact that high frequency modes have increased tendency to reside on the cluster (this is also true for our model, see Fig. 3). The Raman coupling constant of these modes is likely to be enhanced, making the modes visible in the Raman spectrum (more in the experimental samples which have 24\% crystalline fraction, than in the model structure where the fraction is just $10 \%$ ), without visibly distorting VDOS.

Localization properties of the modes can be judged from the participation ratio $p(j)$, which indicates how many atoms "participate" in vibrational eigenmodes $j$. Inverse participation ratio $1 / p$ for $a-\mathrm{Si}$ and $p$-Si, as a function of mode frequency is shown in Fig. 2. The majority of vibrations in both models is delocalized, with the localization transition taking place at around $72 \mathrm{meV}$ (the mobility edge). The modes around $30 \mathrm{meV}$ and some modes below $10 \mathrm{meV}$ appear to be localized too. The latter are resonant modes. The extended modes below about $15 \mathrm{meV}$ are propagons, while all the rest are diffusons (with possibly some longitudinal propagons left ${ }^{30}$ at small frequencies). The localization character in both $a$-Si and $p$-Si models is similar. The presence of the crystalline cluster does not lead to additional localized modes elsewhere in the spectrum. Note that the modes with large weight on the crystalline factor may have character different from diffusons (for example, the modes can resemble propagons in the crystalline regions), so the designation of the Ioffe-Regel limit in $p$-Si should be taken as an indication that the overall character of the modes in the amorphous matrix changes.

In order to understand what fraction of each mode resides 
on the crystalline cluster in the $p$-Si model, we compute the weight each mode has on the cluster (that is, we sum $\left|\mathbf{e}_{a}^{j}\right|^{2}$ for each $j$ over all atoms $a$ from the cluster). The result is shown in Fig. 3, together with a histogram of VDOS of the cluster calculated by solving the dynamical equations for the cluster atoms with the surrounding atoms held fixed. An unbiased mode has a weight of $0.086(8.6 \%)$, corresponding to the percentage of the atoms making up the cluster. For all the modes below the mobility edge the weight fluctuates around 0.086, showing no special affinity for the cluster. Localized modes, as would be expected from their idiosyncratic character, can be localized (fully or partially) on, through, or off the cluster. None of the modes is localized fully on the cluster. There are four locons with the weight on the cluster of $30 \%$ or greater, the maximum weight being that of $59 \%$ for a mode with frequency $\omega=73.05 \mathrm{meV}$ and participation ratio $p=13$. The second most localized mode on the cluster has the frequency of $72.69 \mathrm{meV}$, the weight of $55 \%$ and $p=12$. The third and fourth modes are more delocalized, having frequency (weight, $p$ ) $\omega=70.67(31 \%, 160)$ and $\omega=71.12$ $(30 \%, 117)$, respectively. All four modes lie in the mobility edge region. In addition to these, there are modes with frequencies around $30 \mathrm{meV}$ which have enhanced affinity for the cluster (see Fig. 3). The weight of these modes at the crystalline cluster does not exceed $30 \%$, but six modes have the weight between 20 and $30 \%$.

Harmonic vibrations in $a$-Si explain well many observed thermodynamic properties ${ }^{38}$ of the material, as well as kinetics such as heat flow. ${ }^{20,31}$ Anharmonicity does not directly affect heat flow in dielectric glasses, but is very important in relaxing the perturbed vibrations to maintain local equilibrium (temperature gradient, to be specific). More directly, anharmonicity affects thermal expansion and sound attenuation. The 1000 -atom model of $a$-Si was employed to demonstrate the importance of thermal vibrations in both of these phenomena. ${ }^{39,40}$ It was found that anharmonicity is rather weak in $a-\mathrm{Si}$, although somewhat stronger than in $c$-Si, primarily due to strong anharmonicity of resonant modes. Indeed, resonant modes show giant Grüneisen parameters in the model, strongly enhancing the effects of anharmonicity, although still within the limits of perturbation theory based on cubic anharmonicity.

\section{VIBRATIONAL LIFETIMES}

Using cubic anharmonicity as the small perturbation to the harmonic Hamiltonian, anharmonic decay rate $2 \Gamma(j)$ of mode $j$ can be obtained from the formula ${ }^{41,42}$

$$
\begin{aligned}
2 \Gamma(j)= & \frac{\hbar^{2} \pi}{4 \omega(j)} \sum_{k l} \frac{|V(j, k, l)|^{2}}{\omega(k) \omega(l)}\left(\frac{1}{2}[1+n(k)\right. \\
& +n(l)] \delta[\omega(j)-\omega(k)-\omega(l)] \\
& +[n(k)-n(l)] \delta[\omega(j)+\omega(k)-\omega(l)]) .
\end{aligned}
$$

Here $\omega(j)$ is the frequency of mode $j, n(j)$ is the mode occupation number given by $n(j)=\left\{\exp \left[\hbar \omega(j) / k_{B} T\right]-1\right\}^{-1}$ with $T$ denoting temperature, and $V(j, k, l)$ is the matrix ele- ment of the cubic anharmonicity of the interatomic potential $V$ in the harmonic representation

$$
V(j, k, l)=\sum_{a b c} \sum_{\alpha \beta \gamma} \frac{\partial^{3} V}{\partial u_{a \alpha} \partial u_{b \beta} \partial u_{c \gamma}} \frac{e_{a \alpha}^{j}}{\sqrt{m_{a}}} \frac{e_{b \beta}^{k}}{\sqrt{m_{b}}} \frac{e_{c \gamma}^{l}}{\sqrt{m_{c}}} .
$$

Greek symbols $\alpha, \beta$, and $\gamma$ stand for the Cartesian coordinates of both the atomic displacements $\mathbf{u}$ from the equilibrium positions, and of the normalized vibrational eigenvectors e. The atomic masses are denoted as $m$. Anharmonic vibrational lifetimes are the inverse of the rates

$$
\tau(j)=1 / 2 \Gamma(j) .
$$

In this paper we present decay rates in the units of meV. For conversion into lifetimes, a decay rate of $1 \mathrm{meV}$ is equivalent to a lifetime of about $0.7 \mathrm{ps}$.

In Eq. (1) the term with the temperature factor $1+n(k)$ $+n(l)$ corresponds to the "combination" decay $j \rightarrow k+l$, while the term with $n(k)-n(l)$ represents the "difference" decay $j+k \rightarrow l$. Energy conservation is ensured by the delta functions. At low (down to zero) temperatures the first term in Eq. (1) dominates, giving rise to a constant $2 \Gamma$, while both terms are generally equally important at large temperatures, where $\Gamma \sim T$. In crystals $V(j, k, l)$ vanishes unless the modes' momentum is conserved in the decay process. In glasses, where lattice momentum itself is not a valid concept (except for propagons and resonances), all the modes $\mathrm{k}$ and 1 from the spectrum contribute to $V(j, k, l)$ for a given $j$.

Crucial for determining decay rates perturbatively from a finite-size model is the $\delta$ function regularization. We approximate $\delta(\omega) \approx \theta_{w}(\omega)$, where $\theta_{w}(\omega)$ is a rectangle of width $w$ and height $1 / w$ centered at $\omega=0$. In our calculations with 1000 atoms we choose $w=1 \mathrm{meV}$, which fits about 40 modes in the rectangle. The choice of $w$ needs to be a compromise between good statistics and computer power. The statistics is determined by both the number and "similarity" of the modes in a rectangle. If $w$ is too large, the rectangle function will sample modes with distinct characteristics, not representing faithfully the modes of the chosen frequency. This problem is likely to be absent for diffusons, which do not differ much on small spectral scales due to the absence of degeneracy (cf. Ref. 39), but may be relevant for propagons (which are mixed with resonant modes) and locons (which are idiosyncratic). Fortunately, the averaging, first within the rectangle and second, over the whole spectrum [see Eq. (1)] makes the decay rates quite insensitive to the choice of $w$, for a reasonable interval of values. In the earlier calculation ${ }^{6} w$ was chosen to be $0.4 \mathrm{meV}$ for a 216-atom model, fitting about four modes in the rectangle. As we will see from the comparison of the two calculations in the following section, this choice was already good enough, although it may have contributed somewhat to the statistical noise, especially at low temperatures and small frequencies. To illustrate the effect $w$ has on the decay rates, we show in Fig. 4 the calculated rates of the mode with $\omega=12.49 \mathrm{meV}$ in $a-\mathrm{Si}$, as a function of temperature, for selected $w$, ranging from $0.12 \mathrm{meV}$ (corresponding to about 4 modes per rectangle) to $2.4 \mathrm{meV}$ (80 modes/rectangle). Except for the smallest $w$, the 


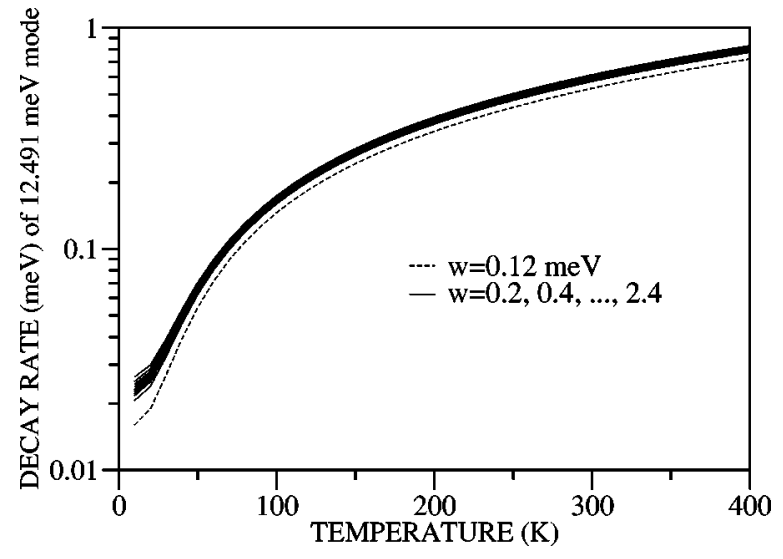

FIG. 4. Calculated decay rate of the mode (a propagon) with frequency $\omega=12.49 \mathrm{meV}$ in $a-\mathrm{Si}$ as a function of temperature for different widths $w$ of the rectangle function $\theta_{w}(\omega)$ representing the delta function in Eq. (1). The curves are for $w$ equal 0.12 (dashed line) and $0.2,0.4, \ldots, 2.4 \mathrm{meV}$ (solid lines), the order of which is not mirrored in the magnitude of the curves. The greatest decay rate is for $w=0.2 \mathrm{meV}$, while the lowest for $w=1.4 \mathrm{meV}$. The curve representing $w=1 \mathrm{meV}$ chosen in the calculation is in the middle of the bunch.

results are grouped together with the dispersion of less than $10 \%$ above $100 \mathrm{~K}$. The greatest dispersion is at the lowest temperatures, where it reaches $25 \%$. (The low temperature properties of the model do not describe well the real $a-\mathrm{Si}$ structure, because of the existence of the minimum frequency of $4 \mathrm{meV}$ in the model). Figure 5 shows the decay rate for the same mode as a function of $w$, for selected temperatures. The rates become reasonably insensitive to $w$ above $0.4 \mathrm{meV}$. The dispersion due to the sensitivity on $w$ is a factor contributing to the uncertainty of the calculated values.

\section{AMORPHOUS SILICON}

We now present the calculated decay rates for the 1000atom model of $a$-Si. Due to the computational power limitations we have sampled the spectrum uniformly with about

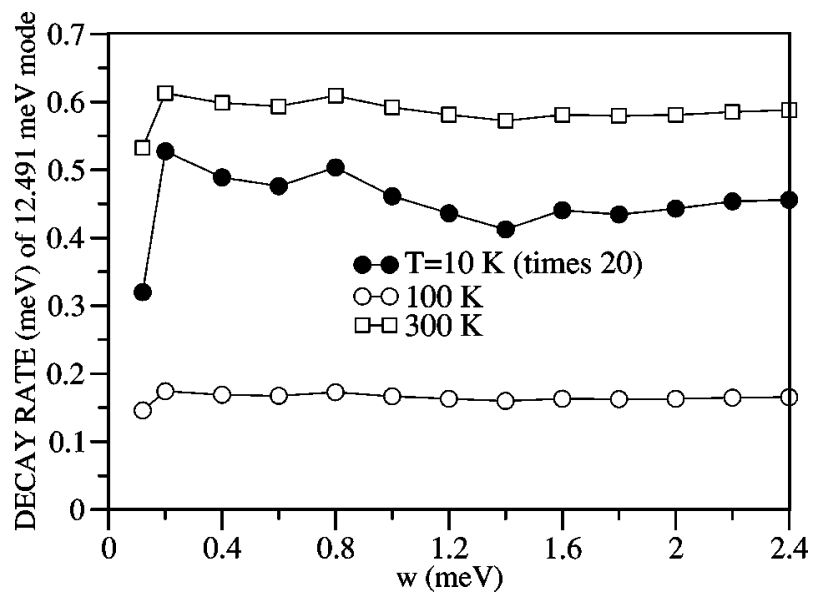

FIG. 5. Calculated decay rate of the mode with frequency $\omega$ $=12.49 \mathrm{meV}$ in $a-\mathrm{Si}$ as a function of $w$ at 10, 100, and $300 \mathrm{~K}$.

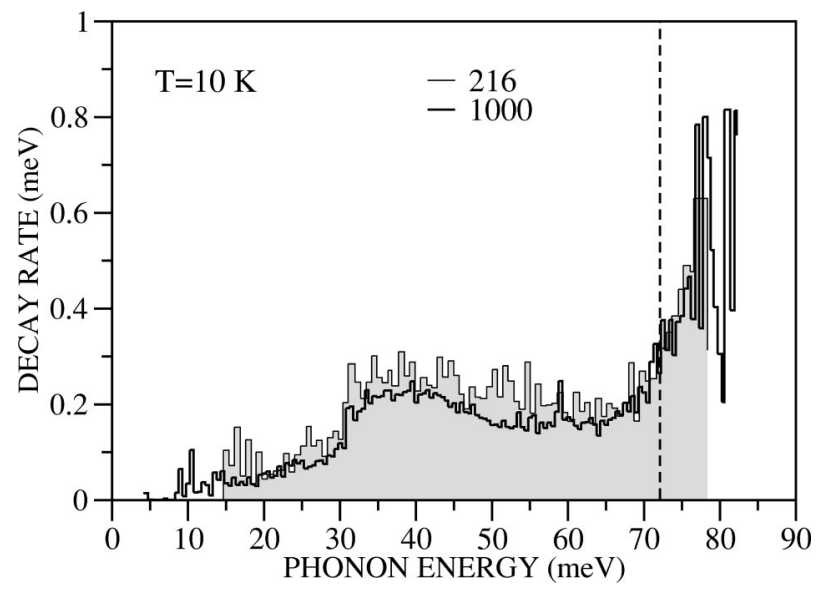

FIG. 6. Calculated decay rates versus frequency for the 1000atom model at $10 \mathrm{~K}$ (thick line). For comparison the rates of the 216-atom model from Ref. 6 (not smoothed) are also shown (shaded area).

200 modes for which we computed $2 \Gamma$. The calculated decay rates are presented as a function of the mode frequency for two different temperatures: $10 \mathrm{~K}$ in Fig. 6 and $300 \mathrm{~K}$ in Fig. 7. For comparison the previous calculations ${ }^{6}$ on a $216-$ atom model of $a$-Si are included. Overall, the decay rates for the two models agree. The rates are on the order of $\mathrm{meV}$ (picosecond lifetimes). Perturbation theory is thus valid for all the sampled modes with the exception of few in the lowest part of the spectrum at $300 \mathrm{~K}$ (see below). As was shown in Ref. 6 the decay rates as a function of frequency at $10 \mathrm{~K}$ (and at low temperatures, generally) follow the joint density of states $\left\{\Sigma_{k l} \delta[\omega(j)-\omega(k)-\omega(l)]\right\}$ which counts, for a chosen mode $j$, the number of combination decay possibilities $j \rightarrow k+l$ with the constraint of energy conservation. At larger temperatures one must add the number of difference decay channels $j \rightarrow k-l$ to reproduce, qualitatively, the calculated $2 \Gamma(\omega)$. These up-conversion processes become important for most diffusons at $T \gtrsim 100 \mathrm{~K}$. More detailed physics of the anharmonic decay in glasses and especially the

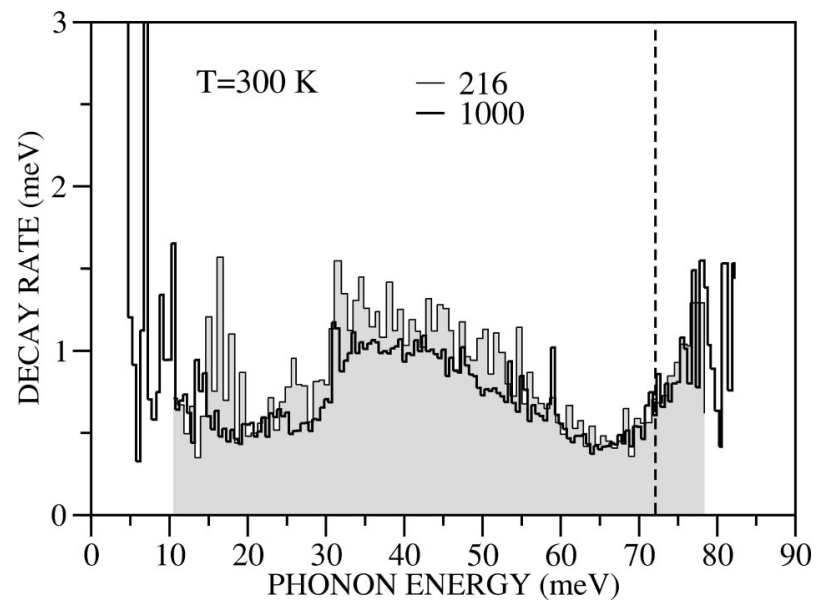

FIG. 7. Calculated decay rates versus frequency for the 1000atom model at $300 \mathrm{~K}$. For comparison the rates of the 216-atom model from Ref. 6 (not smoothed) are also shown (shaded area). 


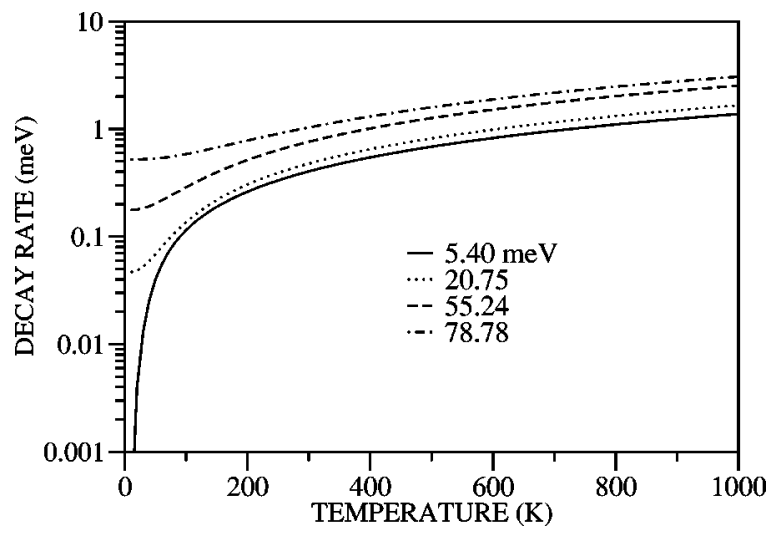

FIG. 8. Calculated decay rates of selected modes in $a$-Si versus temperature. The lines are labeled according to modes' frequencies in $\mathrm{meV}$. The lowest frequency mode is a propagon, the following two are diffusons (acousticlike and opticlike) and the highest frequency mode is a locon.

statistics of the decay matrix elements can be found in Ref. 6.

There are several features which make the calculated decay rates for the 216-atom and 1000-atom models somewhat different. The first is the overall reduction in noise for the 1000 -atom model (the data are not smoothed as was done in Ref. 6). The reason is both the greater model size (spectral averaging) and greater $w$ (rectangle averaging). Note that the observed noise in the spectrally resolved $2 \Gamma$ is about $10 \%$ or less, consistent with a dispersion of the decay rates with $w$, discussed in the previous section. Second, the calculated rates for the 1000-atom model are somewhat smaller than those of the 216-atom model, that is, the latter model appears to be slightly more anharmonic. This is at variance with the calculation of thermal expansion ${ }^{39}$ where the 216-atom model seems less anharmonic. The latter difference probably can be explained by the anomalously large negative mode Grüneisen parameters of the low frequency resonance modes of the 1000 atom model, as the thermodynamic Gruneisen parameter depends on an average mode Gruneisen parameter at high temperatures. We note that the structural models differ in other ways: the smaller model is more topologically constrained, ${ }^{39}$ has smaller energy/atom, and has higher density than the 1000-atom model. Third, the calculated rates of the 1000-atom model extend to a lower frequency region as the minimum frequency of the model is smaller than that of the 216-atom model. Finally, some low-frequency modes (resonances) at $300 \mathrm{~K}$ exhibit giant decay rates, comparable to the modes' frequencies. These rates are in fact invalid, since they are not consistent with perturbation theory. However, they indicate what may be expected from a full anharmonic calculation (for example, by molecular dynamics). This important physics issue will be discussed elsewhere.

In Fig. 8 we plot the temperature dependence of the decay rates of selected modes. We show the temperature dependence for a propagon, an acousticlike and an opticlike diffuson, and a locon. The low-frequency propagon has a divergent lifetime (decay rate vanishes) as temperature decreases to zero, since there are no two modes into which it could decay, due to the energy conservation constraint and the ex-

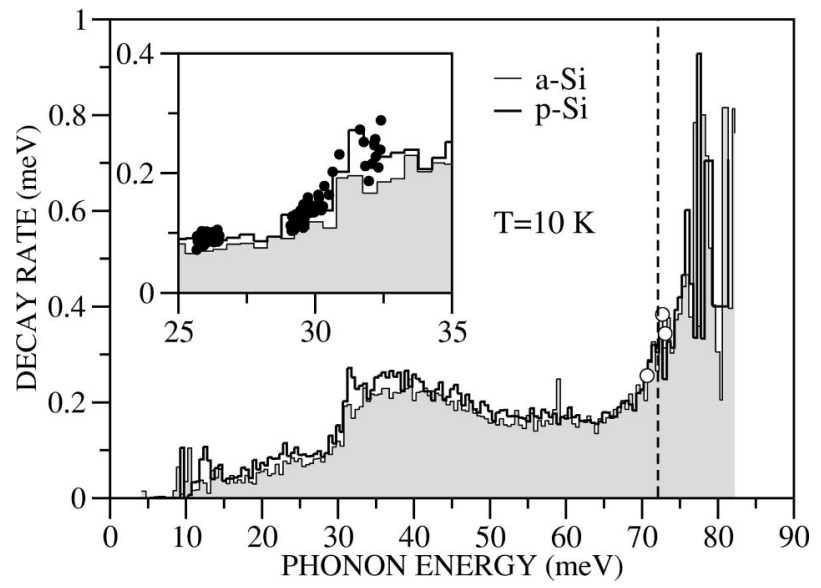

FIG. 9. Calculated decay rates of the 1000-atom model of $p$-Si at $10 \mathrm{~K}$. For comparison, the rates for the 1000-atom model of $a-\mathrm{Si}$ are also shown (shaded area). The empty circles are for three modes in the mobility region with more than $30 \%$ weight on the crystalline cluster, while the inset shows the decay rates (filled circles) of modes around $30 \mathrm{meV}$, which have large affinity (weight up to 30 $\%$ ) for the cluster.

istence of the minimum-frequency mode. All the other modes have constant decay rates at small temperatures. The constant goes smoothly to a linear function at large temperatures, which is due to the fact that the population density of thermal phonons increases linearly with temperature.

\section{PARACRYSTALLINE SILICON}

The results for the 1000-atom model of $p$-Si are shown in Figs. 9 and 10, which plot $2 \Gamma$ as a function of mode frequency. For comparison we also present the data for $\mathrm{a}-\mathrm{Si}$ discussed in the previous section. The results are quantitatively similar for both models. There are no anomalous decay rates appearing in the spectrum of $p$-Si which would be due to the crystalline cluster. In addition to the sampling modes,

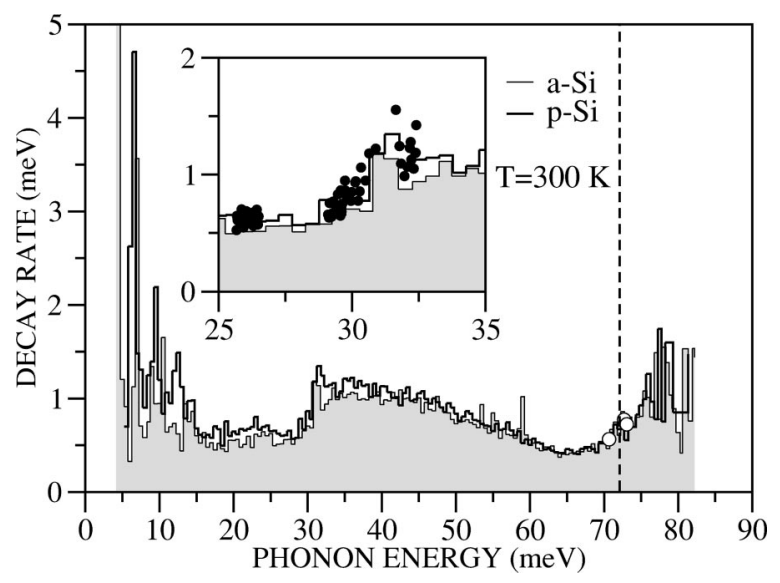

FIG. 10. Calculated decay rates of the 1000-atom model of $p$-Si at $300 \mathrm{~K}$. For comparison, the rates for the 1000-atom model of $a-\mathrm{Si}$ are also shown (shaded area). The empty circles are for three modes with more than $30 \%$ weight on the crystalline cluster, and the inset plots the decay rates (filled circles) of the modes around $30 \mathrm{meV}$ with large weight on the cluster. 


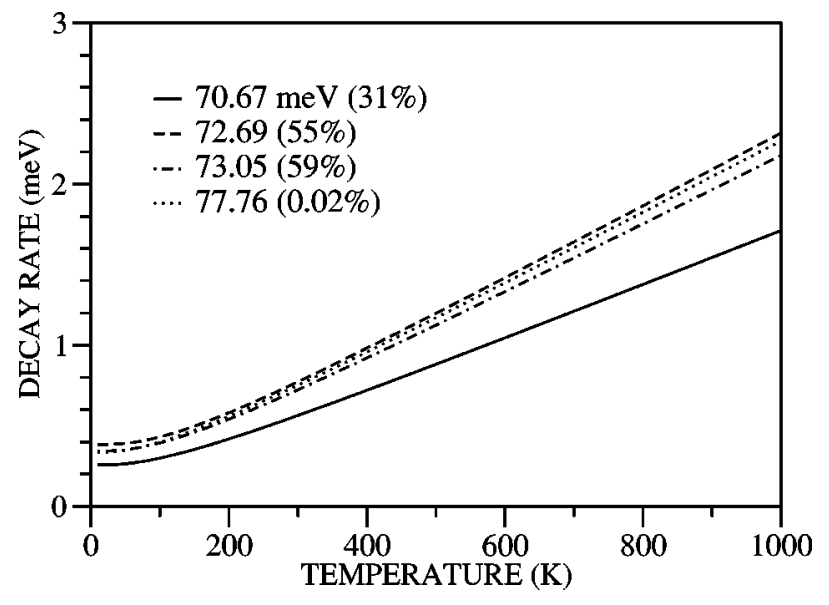

FIG. 11. Calculated decay rates for selected locons in $p$-Si. The curves are labeled according to frequency in $\mathrm{meV}$. The numbers in the brackets show the modes' weight on the crystalline cluster.

we have computed the decay rates specifically for three modes in the mobility edge region with the weight at the crystalline cluster greater than $30 \%$. They are presented in Figs. 9 and 10 by empty circles. The decay rates of these modes have the same magnitude as those of the other locons. Finally, in the insets of the two figures we show the decay rates of modes with frequencies around $30 \mathrm{meV}$, the region of especially high affinity for the cluster (see Fig. 3). Decay rates of more than 80 modes in that spectral region are plotted. Although many of the modes have large weight (some of them up to $30 \%$ ) on the cluster, most are unbiased. The fact that $2 \Gamma$ of all of these modes are similar in magnitude at different temperatures implies no special decay behavior for the modes of strong affinity for the cluster.

Figure 11 shows the temperature dependence of three modes with more than $30 \%$ weight on the crystalline cluster. The modes have frequencies (weight, p) 70,67 meV $(31 \%, 160), 72.68 \mathrm{meV}(55 \%, 12)$, and $73.05 \mathrm{meV}(59 \%, 13)$. In addition, the figure plots the decay rate of a "normal" locon with $\omega=77.76 \mathrm{meV}(0.02 \%, 8)$, residing outside of the cluster. The decay rates are constant at the lowest temperatures, increasing linearly with increasing $T$ at higher temperatures. The mode residing outside the cluster $(77.76 \mathrm{meV})$ has $\Gamma$ of similar magnitude as for the two modes with weight of more than $50 \%$ on the cluster.

Finally, in Fig. 12 we plot the anharmonic matrix elements $V(j, k, l)$ of the combination decay $j \rightarrow k+l$ for the maximally localized mode on the cluster, with frequency $73.05 \mathrm{meV}$ (weight 59\%) to visualize the mode's decay channels. The figure shows that the dominant channel is a decay into two diffusons. Decay into a propagon and a diffuson (the points in Fig. 12 below $15 \mathrm{meV}$ and above 58 $\mathrm{meV}$ ) is somewhat less important; the corresponding matrix elements are much smaller. This may be related to the fact that propagon's weight on the cluster is systematically lower than $8.6 \%$ (see Fig. 3). The diffusons' weight on the cluster is much more scattered, with a significant number of diffusons having the weight of $8.6 \%$ and more. Decay into another locon and a propagon is forbidden by energy conservation. Most importantly, the decay channels are spread over

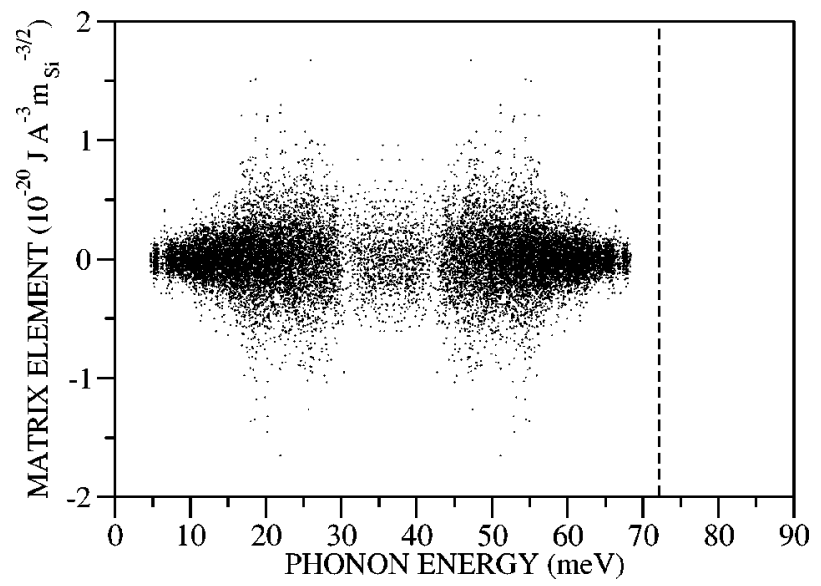

FIG. 12. Calculated matrix element $V(j, k, l)$ of $p$-Si for mode $j$ maximally localized $(59 \%)$ on the crystalline cluster $[\omega(j)$ $=73.05 \mathrm{meV}]$ as a function of $\omega(k)$. Shown are data for $k$ and $l$ obeying energy conservation $|\omega(j)-\omega(k)-\omega(l)|<w / 2$, where $w$ $=0.2 \mathrm{meV}$ (taken to be smaller than $w=1 \mathrm{meV}$ used in the calculation, to get a manageable graphics size).

the whole spectrum, with no anomalous dominating scattering probabilities to few selected modes, which would indicate decoupling of the mode from the amorphous matrix.

\section{CONCLUSIONS}

We have calculated anharmonic decay rates of 1000-atom models of $a$-Si and $p$-Si using perturbation theory with cubic anharmonicity in the interatomic potential. The results for $a$-Si are in agreement with the previous perturbative calculations on a smaller model, as well as with a molecular dynamics calculation. The results reiterate the previous findings that the vibrational lifetimes are on the picosecond time scales, generally increasing with increasing frequency. The decay rates of locons are idiosyncratic, but are by no means inhibited. Calculated decay rates of $p$-Si are similar to those of $a$-Si, showing little sensitivity to structural properties. These findings disagree with the interpretation of recent experiments which find decay rates on the order of nanoseconds and somewhat greater sensitivity to structural properties.

The explanation that we offer to account for these discrepancies is that the calculation and experiment refer to two different things. First, as we have pointed out earlier, simple (and at such scale usually over-relaxed) models such as a continuous random network type WWW model or a similar model containing a crystalline grain used in this study cannot faithfully reproduce a broad range of various topological features-some or combinations of which may be responsible for increased decay times observed in the experimentpresent in a real material. Second, in our calculations only "perturbative" decay rates, where a small (infinitesimal) population of a single mode goes out of equilibrium are computed. The experiments measure the decay of vibrational states excited over a large portion of the spectrum. Furthermore, the laser excitation produces phonon populations too far off the equilibrium to be called small perturbations. In Ref. 3, for example, the excited phonon population $n$ lies 
between 0.03 and 0.3 , which, for a mode with frequency, say, $50 \mathrm{meV}$ corresponds to an effective temperature of 160 and $400 \mathrm{~K}$, respectively. This is huge compared to $2 \mathrm{~K}$ at which the samples are held. A numerical investigation of Bickham ${ }^{8}$ indeed shows that a strong perturbation of the vibrational spectrum of $a$-Si can relax on a 100 ps time scale, compared to $10 \mathrm{ps}$ for a weak perturbation. In addition to pure vibrational relaxation, it is also likely, as suggested by Bickham and Feldman, ${ }^{7}$ that correspondingly large local deviations in the atomic displacements cause local structural rearrangements which may relax to local metastable minima while emitting phonons. It is possible that these rearrangements occur on nanosecond time scales. This effect would decrease in time as the perturbed structure progresses through deeper local minima.

We conclude that current simple models for $a$-Si in combination with presented above methods of analysis do not provide an answer to the question why vibrations in real material under the specific experimental conditions appear to decay over such long time scales. A better answer may be obtained by studying larger and more realistic models when the required computational power becomes available. However, considering the consistent results obtained thus far with different models (of sizes from 216 to 4096 atoms) and different techniques (perturbation theory and molecular dynamics) it is rather unlikely that the interpretation of the experimental findings in terms of the (pure) vibrational lifetimes (that is, without considering structural relaxation and possibly photoexcited-electron recombination processes) will be validated by investigating larger models.

\section{ACKNOWLEDGMENTS}

We thank Phil Allen for useful discussions and M. van der Voort for suggesting this calculation.
${ }^{1}$ A. Scholten, A. V. Akimov, and J. I. Dijkhuis, Phys. Rev. B 47, 13910 (1993).

${ }^{2}$ A. Scholten and J. I. Dijkhuis, Phys. Rev. B 53, 3837 (1996).

${ }^{3}$ M. van der Voort, A. V. Akimov, and J. I. Dijkhuis, Phys. Rev. B 62, 8072 (2000).

${ }^{4}$ M. van der Voort, O. L. Muskens, A. V. Akimov, A. B. Pevtsov, and J. I. Dijkhuis, Phys. Rev. B 64, 045203 (2001).

${ }^{5}$ J. Menendez and M. Cardona, Phys. Rev. B 29, 2051 (1984).

${ }^{6}$ J. Fabian and P. B. Allen, Phys. Rev. Lett. 77, 3839 (1996).

${ }^{7}$ S. R. Bickham and J. L. Feldman, Phys. Rev. B 57, 12234 (1999).

${ }^{8}$ S. R. Bickham, Phys. Rev. B 59, 4894 (1999).

${ }^{9}$ S. Alexander, C. Laermans, R. Orbach, and H. M. Rosenberg, Phys. Rev. B 28, 4615 (1983).

${ }^{10}$ A. Jagannathan, R. Orbach, and O. Entin-Wohlman, Phys. Rev. B 39, 13465 (1989).

${ }^{11}$ R. Orbach and A. Jagannathan, J. Phys. Chem. 98, 7411 (1994).

${ }^{12}$ J. Fabian, Phys. Rev. B 55, 3328 (1997).

${ }^{13}$ D. Leitner, Phys. Rev. B 64, 094201 (2001).

${ }^{14}$ D. Leitner, Phys. Rev. Lett. 87, 188102 (2001).

${ }^{15}$ S. M. Nakhmanson, P. M. Voyles, N. Mousseau, G. T. Barkema, and D. A. Drabold, Phys. Rev. B 63, 235207 (2001).

${ }^{16}$ P. M. Voyles, J. E. Gerbi, M. M. J. Treacy, J. M. Gibson, and J. R. Abelson, Phys. Rev. Lett. 86, 5514 (2001).

${ }^{17}$ F. Wooten, K. Winer, and D. Weaire, Phys. Rev. Lett. 54, 1392 (1985).

${ }^{18}$ G. T. Barkema and N. Mousseau, Phys. Rev. Lett. 77, 4358 (1996)

${ }^{19}$ N. Mousseau, G. T. Barkema, and S. M. Nakhmanson, Philos. Mag. B 82, 171 (2002).

${ }^{20}$ J. L. Feldman, M. D. Kluge, P. B. Allen, and F. Wooten, Phys. Rev. B 48, 12589 (1993).

${ }^{21}$ F. H. Stillinger and T. A. Weber, Phys. Rev. B 31, 5262 (1985).

${ }^{22}$ R. Biswas, A. M. Bouchard, W. A. Kamikatahara, G. S. Grest, and
C. M. Soukolis, Phys. Rev. Lett. 60, 2280 (1988).

${ }^{23}$ H. R. Schober and B. B. Laird, Phys. Rev. B 44, 6746 (1991).

${ }^{24}$ H. R. Schober and C. Oligschleger, Phys. Rev. B 53, 11469 (1996).

${ }^{25}$ S. N. Taraskin and S. R. Elliott, Phys. Rev. B 61, 12031 (2000).

${ }^{26}$ S. N. Taraskin and S. R. Elliott, Phys. Rev. B 61, 12017 (2000).

${ }^{27}$ M. Marinov and N. Zotov, Phys. Rev. B 55, 2938 (1999).

${ }^{28}$ P. B. Allen, J. L. Feldman, J. Fabian, and F. Wooten, Philos. Mag. B 79, 1715 (1999).

${ }^{29}$ E. Courtens, M. Foret, B. Hehlen, and R. Vacher, Solid State Commun. 117, 187 (2001).

${ }^{30}$ J. L. Feldman, J. Non-Cryst. Solids 307-310, 128 (2002).

${ }^{31}$ P. B. Allen and J. L. Feldman, Phys. Rev. B 48, 12581 (1993).

${ }^{32}$ M. Foret, B. Hehlen, G. Taillades, E. Courtens, R. Vacher, H. Casalta, and B. Dorner, Phys. Rev. Lett. 81, 2100 (1998).

${ }^{33}$ G. Ruocco, F. Sette, R. D. Leonardo, D. Fioretto, M. Krisch, M. Lorenzen, C. Masciovecchio, G. Monaco, F. Pignon, and T. Scopigno, Phys. Rev. Lett. 83, 5583 (1999).

${ }^{34}$ O. Pilla, A. Cunsolo, A. Fontana, C. Masciovecchio, G. Monaco, M. Montagna, G. Ruocco, T. Scopigno, and F. Sette, Phys. Rev. Lett. 85, 2136 (2000).

${ }^{35}$ E. Rat, M. Foret, E. Courtens, R. Vacher, and M. Arai, Phys. Rev. Lett. 83, 1355 (1999).

${ }^{36}$ M. Foret, R. Vacher, E. Courtens, and G. Monaco, Phys. Rev. B 66, 024204 (2002).

${ }^{37}$ R. L. C. Vink, G. T. Barkema, W. F. van der Weg, and N. Mousseau, J. Non-Cryst. Solids 282, 248 (2001).

${ }^{38}$ J. L. Feldman, C. S. Hellberg, G. Viliani, W. Garber, and F. M. Tangerman, Philos. Mag. B 82, 133 (2002).

${ }^{39}$ J. Fabian and P. B. Allen, Phys. Rev. Lett. 79, 1885 (1997).

${ }^{40}$ J. Fabian and P. B. Allen, Phys. Rev. Lett. 82, 1478 (1999).

${ }^{41}$ A. A. Maradudin and A. E. Fein, Phys. Rev. 128, 2589 (1961).

${ }^{42}$ R. A. Cowley, Adv. Phys. 12, 421 (1963). 